\title{
Direct injection of anti-cancer drugs into endobronchial tumours for palliation of major airway obstruction
}

\author{
Seyhan I Çelikoğlu, Tuncer Karayel, Sabriye Demirci, Firuz Çelikoğlu, Tülin Çağatay
}

\begin{abstract}
Summary
Patients with tracheal or major airway obstruction due to inoperable carcinomas are at a high risk of developing respiratory failure or post-obstructive pneumonia, or both. This often leads to death in days or weeks. In such cases there is usually an urgent need to restore the airway. This report details the short-term results and techniques used for the treatment of airway obstruction by direct intratumoural injection of several anti-cancer drugs. A total of 93 patients with nearly complete extrinsic obstruction of at least one major airway were treated by injection of anti-cancer drugs directly into the endobronchial tumours or infiltrated bronchial mucosa through a flexible fiber-optic bronchoscope. At every session of treatment 1$3 \mathrm{ml}$ each of $50 \mathrm{mg} / \mathrm{ml}$ 5-fluorouracil, $1 \mathrm{mg} /$ $\mathrm{ml}$ mitomycin, $5 \mathrm{mg} / \mathrm{ml}$ methotrexate, $10 \mathrm{mg} / \mathrm{ml}$ bleomycin and $2 \mathrm{mg} / \mathrm{ml}$ mitoxantrone were injected separately at different sites without pre-mixing.

Local intratumoural chemotherapy relieved the obstruction in 81 of the 93 patients. Endoscopically visible tumours were reduced in size, and infiltrative changes were also improved. Obstruction was not relieved in 12 patients. The therapy was well tolerated and had no systemic side-effects, and no serious complications. Intratumoural chemotherapy can be considered a new life-saving palliative method in patients with life-threatening airway obstruction.
\end{abstract}

Keywords: bronchoscopy, cytostatic therapy, airway obstruction, injection techniques, chemotherapy, endobronchial tumour

Obstruction of the central airways, either at the time of initial presentation, or more commonly by tumours recurring after initial therapy, is a serious problem. Patients with tracheal or major airway obstruction due to inoperable carcinoma are at a high risk of developing respiratory failure or post-obstructive pneumonia, which often lead to death by slow asphyxiation in days or weeks. In these cases there is usually an urgent need to restore the patency of the air passage.

Patients with symptomatic airway obstruction persisting or developing after radiotherapy or systemic chemotherapy were unsuitable for further treatment with these conventional methods. Moreover, in patients with newly diagnosed inoperable carcinomas, the conventional methods of cancer treatment do not usually relieve the obstruction fast enough. There is, therefore, a place for new methods of treatment of bronchial carcinoma, even if these are only palliative, provided that they are well tolerated and have few side-effects. Photoresection of central obstructing malignant neoplasms of the tracheobronchial tree with $\mathrm{Nd}$ YAG lasers has been used with some success for this purpose. ${ }^{1-5}$

Wagai et $a l .{ }^{6}$ reported that by injecting several anti-cancer drugs directly into the endobronchial tumours or infiltrated mucosa through a fiber-optic bronchoscope, tumour mass was reduced in size, and the accompanying atelectasis or obstructive pneumonia were improved. Their results suggested that this form of therapy might be a useful alternative for the palliation of tracheobronchial obstruction due to malignant tumour. This report details the short-term results and techniques used in 93 patients with major airway obstruction treated by direct intratumoural injection of several anti-cancer drugs.

\section{Patients and methods}

All patients in this series have been evaluated by the Broncho-Pulmonary Section of the Medical Department of Cerrahpaşa Medical School Hospital of Istanbul University since July 1986. All patients were classified as nonresectable surgically by localisation or by extent of disease or due to poor overall general health. In total, 93 patients (six women, 87 men) aged $26-85$ years; mean age $55.5 \pm 11$ with nearly complete (more than $50 \%$ ) exophytic obstruction of at least one major airway were included in this study. Patients with isolated lobar obstruction were included only if it was believed to contribute to respiratory insufficiency or post-obstructive pneumonia. Patients with obstruction of a more peripheral bronchus were not included in this study. We included only patients in whom metastatic disease was present if the dominant symptom was breathlessness. There was no age limit, and all cell types of tumour were accepted.

Two groups of patients were evaluated as follows: 
Group 1, intratumoural treatment first: 68 patients had bronchogenic carcinoma which produced severe dyspnoea and clinical evidence of severe airflow obstruction or obstructive pneumonia. None had received radiation therapy or systemic chemotherapy before they were treated by injection of anti-cancer drugs. Their treatment plan was intratumoural chemotherapy to open the airway, followed by radiation therapy, and/or chemotherapy.

Group 2, recurrent malignancies: radiation therapy and/or systemic chemotherapy first. 25 patients in group 2 had recurrent carcinoma which had been treated previously by radiation therapy and/or systemic chemotherapy.

The ethical committee of Cerrahpaşa Medical School gave approval for this study. Informed consent specifying the nature of this therapy was obtained from each patient before treatment started.

Preliminary studies included a complete history and physical examination, posteroanterior and lateral chest X-rays, full blood cell count, prothrombin time, platelet count, spirometry and flow volume loops, and arterial blood gas analysis. The protocol of symptom scores, X-ray examinations, physiological tests and blood examinations were repeated every week during initial treatment, and before all subsequent bronchoscopies.

The extent of tumour seen at bronchoscopy was recorded on charts of the bronchial tree, and a microvideo camera (Olympus) was used for video documentation before and after all treatments for further comparative assessment. Computed tomography (CT) of the chest and abdomen with contrast infusion and rapid sequence imaging was employed to display the relationship between the involved bronchus and the surrounding structures, particularly the large intrathoracic blood vessels. Histologically, there were 71 squamous cell carcinomas, 14 small cell carcinomas, two adenocarcinomas, and one adenocystic carcinoma, as well as three other types of carcinoma.

After injecting $5 \mathrm{mg}$ of diazepam intramuscularly, local anaesthesia with $2 \%$ xylocaine was used to numb the pharynx and larynx.
With the patient in sitting position, the flexible fiber-optic bronchoscope (Olympus 1T10 and BF20d) was inserted transnasally into the trachea. Direct intratumoural injection was performed with a flexible needle (Olympus injector NM 8L, NM9L). Injection was made at the site of the endobronchial lesions. When the lesion was seen as a tumour in the bronchus, we selected as the site of the injection the tumour itself or the surrounding bronchial mucosa. When an infiltrating lesion was present, we injected anti-cancer drugs into the infiltrated mucosa (figure, A).

Injection of cytotoxic drugs intratumourally produced an immediate effect which led to necrosis of the tumour. One of the additional benefits of intratumoural injection of anticancer drugs is to provide a haemostatic effect on tumoural tissue, thus allowing piecemeal resection by forceps. Indeed, after intratumoural injections of anti-cancer drugs, a whiteyellow gel of necrotic tumour debris has been observed attached to the injected portion of the tumour or infiltrated mucosa. This material could easily be cleared with suction and by piecemeal resection using forceps, either at the same bronchoscopy session or during subsequent sessions (figure, B). The necrotic tumour debridement also allowed us to inject anticancer drugs more deeply into the tumour mass and infiltrated bronchial wall (figure, $\mathrm{C}$ ).

Initially, intratumoural injections were given at one- to three-day intervals until airway patency could be restored. The average number of treatments required to achieve symptomatic response in a two-week period in 81 patients was four (range $1-6$ ). In patients with recurrent malignancies showing an initial response to this treatment, direct injection was carried out once a week for six to 10 weeks. If the patency of the airways was maintained, these intervals were increased to one or three months. Intratumoural chemotherapy was abandoned if the patency of the airways could not be established by a maximum of six successive sessions of injections and debridement with piecemeal resection, or when the severity of metastatic disease outweighed the potential benefits of continued treatment.

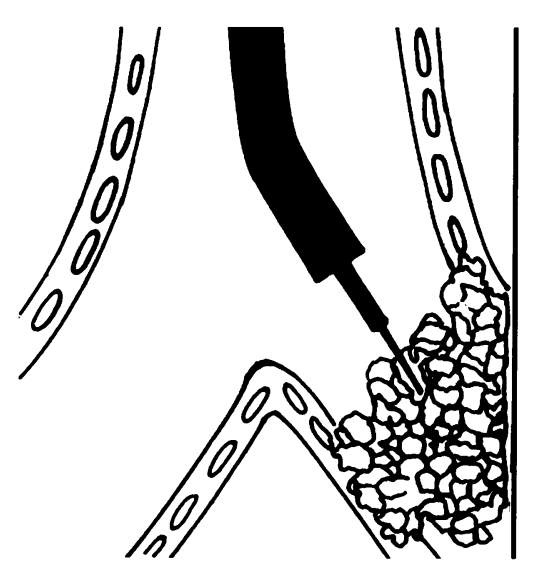

A

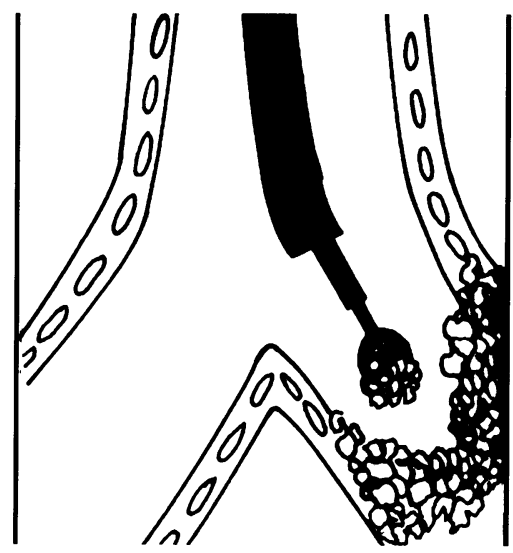

B

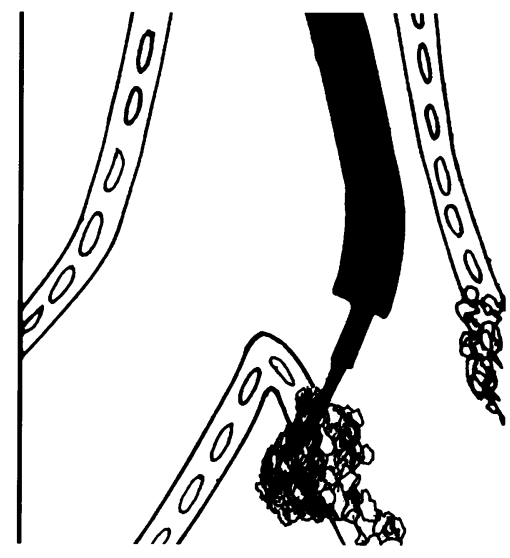

C

Figure (A) Intratumoural injections of anti-cancer drugs; (B) necrotic tumour debridement with foreign body forceps; (C) injection of anticancer drugs more deeply into the tumoural mass and infiltrated mucosa after debridement. 
Criteria for evaluation of therapeutic effects

- good response: more than $50 \%$ increase in calibre of the lumen

- partial response: $25-50 \%$ increase in the calibre of the lumen

- no response: less than $25 \%$ increase in the calibre of the lumen

Box

In 10 patients with obstruction of the trachea or both main bronchi, breathlessness was increased shortly after application of the intratumoural injection because of accumulation of necrotic tumour coagulum and secretions. We performed bronchoscopy immediately and removed necrotic material with the piecemeal resection by foreign body forceps or suction. This restored the patency of the airway.

At every session, according to the size of the tumour, $1-3 \mathrm{ml}$ each of $50 \mathrm{mg} / \mathrm{ml} 5$ fluorouracil, $1 \mathrm{mg} / \mathrm{ml} \mathrm{mitomycin,} 5 \mathrm{mg} / \mathrm{ml}$ methotrexate, $\quad 10 \mathrm{mg} / 1$ bleomycin and $2 \mathrm{mg} / \mathrm{ml}$ mitoxantrone were injected into the tumour separately at different sites without pre-mixing. Drugs were selected which did not induce local necrotic changes in the normal mucosa, which had a $\mathrm{pH}$ of approximately 7.4 and direct neoplastic activity. Drugs which require activation by hepatic microsomes before antineoplastic activity is present (eg, cyclophosphamide), were considered unsuitable for direct injection. The use of varied drugs is justified by their effect on different phases of the cell cycle, thus increasing their synergic effects, preventing development of resistance and using small doses of each to minimise adverse effects.

In this study, the evaluation of therapeutic effects was expressed as the increase of the calibre of the lumen observed in bronchoscopic observation at the end of two weeks of intratumour treatment.

The criteria for evaluation of therapeutic effects are given in the box.

\section{Results}

When tumours were visible as a polypoid mass through a fiber-optic bronchoscope, there was a reduction in size and appearance and atelectasis or obstructive pneumonia were improved after therapy. In six cases airway patency was improved after only a single intratumour treatment and extensive debridement of necrotic material. When tumours infiltrated into the bronchial mucosa, the infiltrative changes were improved markedly by direct injection into the mucosa. When only a small part of the tumour was visible through a fiber-optic bronchoscope, the therapy was useful in decreasing the size of the entire tumour, not just the visible part. This suggests that anti-cancer drugs injected through a fiberoptic bronchoscope may infiltrate deeply into the tumour.

After intratumour chemotherapy, stridor or dyspnoea, post-obstructive pneumonia and haemophthysis were either relieved or completely disappeared in the majority of patients.

In the bronchoscopic examination of 13 of 93 patients there were partial obstructions of the trachea. Involvement of carina with complete obstruction in one of the main bronchi and partial obstruction in the other was observed in four patients. There were 49 patients with obstruction of one of the main bronchi, and 27 patients had an obstruction in one of the lobar bronchi.

The results of obstruction relief are shown in the table. Radiologically, out of 35 patients showing complete obstruction with total collapse of one lung, 18 cases of obstruction were relieved completely and re-expansion fully achieved; in six cases partial re-expansion was observed; and in 11 cases there was no change.

Spirometry was performed successfully before and after intratumoral chemotherapy in 25 patients with main bronchus obstruction. Forced vital capacity (FVC) improved in 20 of the 25 patients (mean $F V C=354.4 \mathrm{ml}$, range $0-1950 \mathrm{ml}$ ). Forced expiratory volume in one second $\left(\mathrm{FVE}_{1}\right)$ improved in 18 of the 25 patients (mean $\mathrm{FEV}_{1}=315.2$, range $0-$ $1650 \mathrm{ml}$ ). Spirometric values improved in 13 of the 22 patients with lobar bronchus obstruction in whom measurements could be obtained before and after treatment (mean FVC $=312.8$, range $0-1250 \mathrm{ml}$; mean $\mathrm{FEV}_{1}=279.2$, range $0-1760 \mathrm{ml}$ ).

Adverse effects were as follows: eight out of 93 patients became slight febrile on the day following intralesional injection. No patient suffered from interstitial pneumonitis, bonemarrow suppression, worsening of inflammatory findings, nausea or depilation. There was no pain or discomfort during intralesional injection of any of the anti-cancer drugs.

Table Overall results of intratumoural chemotherapy in 93 patients

\begin{tabular}{|c|c|c|c|c|c|c|c|c|c|c|}
\hline \multirow{2}{*}{$\begin{array}{l}\text { Airway } \\
\text { localisation }\end{array}$} & \multicolumn{3}{|c|}{ Good response } & \multicolumn{3}{|c|}{ Partial response } & \multicolumn{3}{|c|}{ No response } & \multirow[b]{2}{*}{ Total } \\
\hline & G1 & $G 2$ & $T$ & G1 & G2 & $T$ & G1 & G2 & $T$ & \\
\hline Trachea & 7 & 2 & 9 & 3 & 1 & 4 & - & - & - & 13 \\
\hline Carina + main bronchi & - & - & - & 3 & 1 & 4 & - & - & - & 4 \\
\hline Main bronchus & 14 & 8 & 22 & 20 & 3 & 23 & 2 & 2 & 2 & 49 \\
\hline Lobar bronchus & 5 & 3 & 8 & 8 & 3 & 11 & 7 & 1 & 8 & 27 \\
\hline Total & 26 & 13 & 39 & 34 & 8 & 42 & 9 & 3 & 12 & 93 \\
\hline
\end{tabular}

Abbreviations: G1 (Group 1)= Patient not treated previously; G2 (Group 2)= recurrent carcinoma treated previously by conventional methods; $\mathrm{T}=$ total (For response criteria, see box) 


\section{Discussion}

Laser therapy for palliation of malignant airway obstruction is becoming standard clinical practice at many large medical centres. Intratumoural injection was first developed at Tokyo Medical College in Japan in the early $1970 \mathrm{~s}^{7}$ In this study, the direct injection of several anti-cancer drugs into endobronchial tumours or infiltrated mucosa was used for the same indications as laser applications.

Anti-cancer drugs injected directly into the tumour reduced the size of the intraluminal mass. In most cases, injection of cytotoxic drugs produced an immediate effect which led to necrosis of the tumour. Development of necrosis could be explained by the fact that with direct injection very high concentrations of cytotoxic drugs can be obtained around the tumour cells. Cytotoxic drugs also had some haemostatic effect, probably due to constriction of small vessels in or around the tumour mass. Therefore, after injection of cytotoxic drugs, removal of the tumour mass with forceps was possible, at the same bronchoscopy session. Tumour debridement also allowed us to inject anti-cancer drugs more deeply into the tumour mass and to infiltrate the bronchial wall. It would seem more logical to remove as much tumour as possible by blunt dissection first, and then inject more deeply into the tumour. As blunt dissection usually results in haemorrhage, however, in bulky tumours we first injected cytotoxic drugs, producing a haemostatic effect, then removed the tumour mass with forceps, and injected cytostatic drugs more deeply.

The debridement of necrotic tumour residues with forceps can often re-establish airway patency following even a single session of treatment, relieving stridor or dyspnoea, postobstructive pneumonia and haemoptysis. This manner of treatment has been especially useful for palliation in patients with symptomatic airway obstruction after standard methods of

1 Dumon JR, Shapshay S, Bourecereu J, et al. Neodymium YAG laser in bronchology. Chest 1984; 86:163-8.

2 Dumon JR, Reboud E, Garbe L, Aucomte F, Meric BB. Treatment of tracheobronchial lesions by laser photoresection. Chest 1982; 81: 278-84.

3 Hertzel MR, Millard FJC, Ayesh K. Laser treatment for carcinoma of the bronchus. BMf 1983; 286: 12.

4 Eichenhorn MS, Kvale PA, Miks MM, Seydel HG, Horowitz B, Radke JR. Initial combination therapy with YAG laser photoresection and irradiation for inoperable YAG laser photoresection and irradiation for inoperable
non-small cell carcinoma of the lung. Chest 1986; 80: 782-

5 Joyn

5 Joyner L, JR, Maran AG, Sarama R, Yakaboski A. Neodymium-AG laser treatment of intrabronchial lesions: a new mapping technique via the flexible fiberoptic bronchoscope. Chest 1985; 87: 418-27. therapy such as radiotherapy and systemic chemotherapy have failed. Newly diagnosed patients with symptomatic tracheobronchial obstruction due to inoperable endobronchial carcinoma were also treated initially by direct injection of anti-cancer drugs into the tumour followed by conventional radiotherapy or systemic chemotherapy.

In this series, airway calibre was improved markedly or partially in 81 of 93 patients treated by intratumoural injections over two weeks. Shrinkage of the tumour could be observed immediately after the injections. When there are multiple areas of involvement such as trachea, carina, and main bronchi, injection in each site restores airway patency, even in very severely obstructed cases.

No important adverse effects were observed in relation to intratumour injection. From the viewpoint of the patients the discomfort caused by direct injection of drugs into the tumour is similar to that experienced on a routine fiberoptic bronchoscopy.

Our results show that direct injections of anti-cancer drugs into the tumour are effective in providing immediate symptomatic relief from airway obstruction. Whether or not patient survival is improved is more difficult to answer because the severity of symptoms of patients does not allow placebo-controlled trials.

This study has been extended to allow for the combination of intratumour chemotherapy and conventional radiotherapy in inoperable bronchogenic carcinoma. Intratumour chemotherapy and piecemeal resection of necrotic residue is used initially to eliminate as much as possible of the intrabronchial tumour. Conventional radiation therapy is then given to the same site and to other areas as judged necessary by the radiotherapist.

In this report we present only short-term results obtained in a two-week period. The long-term results are under study and will be the subject of another publication.

6 Wagai F, Kinoshita R, Watanabe RSH, Kitamura S. The direct injection of several anti-cancer drugs into the primary lung cancer through a fiberoptic bronchoscope. In: Nandi PI, Lam WK, eds, Proceedings of seventh Asia Pasific Congress on diseases of the chest. Tokyo: University of Tokyo Faculty of on diseases of the chest. Tokyo:
Medicine, 1981; pp448-50.

7 Hayata Y, Ohbo K, Ogawa I, Taira O. Immunotherapy for lung cancer cases using BCG or BCG cell-wall skeleton: intratumoral injections. Gann Monogr Cancer Res. 1978; 21: 